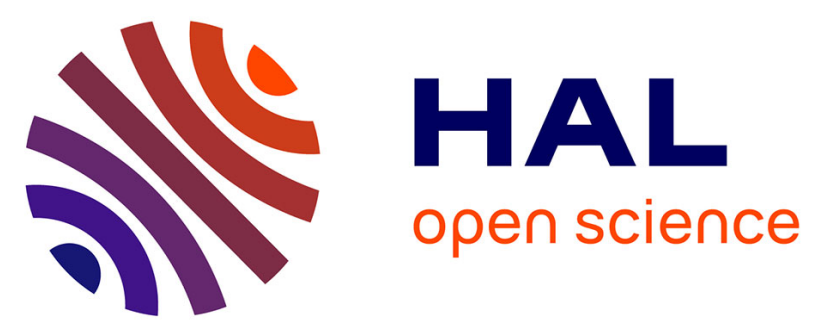

\title{
Topographie, composition et analyse à l'échelle nanoscopique des plaques de Randall débutantes
}

E. Letavernier, D. Bazin, L. Huguet, O. Stephan, A. Gloter, M.C. Verpont, V. Frochot, J.P. Haymann, I. Brochériou, O. Traxer, et al.

\section{- To cite this version:}

E. Letavernier, D. Bazin, L. Huguet, O. Stephan, A. Gloter, et al.. Topographie, composition et analyse à l'échelle nanoscopique des plaques de Randall débutantes. Premier congrès de la Société francophone de néphrologie dialyse et transplantation (SFNDT), Sep 2016, Strasbourg, France. pp.355-356, 10.1016/j.nephro.2016.07.192 . hal-01371625

\section{HAL Id: hal-01371625 \\ https://hal.sorbonne-universite.fr/hal-01371625}

Submitted on 27 Sep 2016

HAL is a multi-disciplinary open access archive for the deposit and dissemination of scientific research documents, whether they are published or not. The documents may come from teaching and research institutions in France or abroad, or from public or private research centers.
L'archive ouverte pluridisciplinaire HAL, est destinée au dépôt et à la diffusion de documents scientifiques de niveau recherche, publiés ou non, émanant des établissements d'enseignement et de recherche français ou étrangers, des laboratoires publics ou privés. 


\author{
Topographie, composition et analyse à l'échelle nanoscopique des plaques de Randall \\ débutantes \\ E. Letavernier ${ }^{1}$, , D. Bazin ${ }^{2}$, L. Huguet ${ }^{3}$, O. Stephan ${ }^{4}$, A. Gloter ${ }^{4}$, M.C. Verpont ${ }^{3}$, V. Frochot ${ }^{5}$, J.P. Haymann ${ }^{5}$, I. \\ Brochériou $^{6}$, O. Traxer ${ }^{7}$, M. Daudon ${ }^{5}$, \\ 1 Explorations fonctionnelles multidisciplinaires, université Paris-Sorbonne/Inserm UMR S 1155/AP-HP, Paris, \\ France \\ 2 Équipe Smile, laboratoire de la chimie de la matière condensée de Paris, Paris, France \\ 3 UMRS 1155, UMR S 1155 Tenon, Paris, France \\ 4 Laboratoire de physique des solides, Orsay, France \\ 5 Explorations fonctionnelles multidisciplinaires, hôpital Tenon, Paris,France \\ 6 Anatomie et cytologie pathologiques, hôpital Tenon, Paris, France \\ 7 Urologie, hôpital Tenon, Paris, France \\ *Auteur correspondant. Adresse e-mail : emmanuel.letavernier@aphp.fr, (E. Letavernier)
}

\title{
Introduction
}

Alexander Randall a identifié dans les années 1930 des dépôts de phosphate de calcium à la pointe des papilles rénales comme étant à l'origine des calculs rénaux oxalo-calciques : les plaques de Randall. Huit décennies plus tard, nous observons à un nombre croissant de calculs générés à partir de ces plaques. Nous avons considéré que l'analyse de plaques « débutantes» pourrait permettre de déterminer leur origine.

\section{Matériels et méthodes}

Cinquante-quatre papilles saines, issues de 22 pièces de néphrectomie réalisées pour cancer, ont été analysées par immunohistochimie couplée à la coloration de Von Kossa ou de Yasue, par microscopie électronique à balayage couplée à des cartographies élémentaires (EDX), par microspectroscopie infrarouge à transformée de Fourier, par cryo-microscopie électronique à transmission couplée à des analyses de perte d'énergie d'électron (cartographies élémentaires) et des techniques de diffraction.

\section{Résultats}

Des plaques de Randall « débutantes » ont été observées dans 72,7\% des reins et près de deux tiers des papilles analysées.Les principaux composants de ces plaques étaient de l'apatite carbonatée, comme attendu, mais des quantités importantes de phosphate de calcium amorphe et de whitlockite, un phosphate mixte calcique et magnésien, ont été retrouvés. Ces plaques débutantes se trouvaient exclusivement à la pointe des papilles, autour de la partie profonde des anses de Henlé $(62,4 \%$ des calcifications) et fréquemment autour des vasa recta $(37,2$ $\%$ des calcifications), mais très rarement autour des tubes collecteurs. L'analyse à l'échelle nanoscopique de ces microcalcifications a révélé différents types de structures, notamment des nanocristaux contenus dans des vésicules mesurant quelques dizaines à quelques centaines de nanomètres.

\section{Discussion}

Ces résultats mettent en évidence un processus de calcification généré dans l'interstitium et non dans les tubules, avec un rôle évident des vasa recta dans leur genèse. La composition variée de ces plaques, leur contenu en magnésium ou l'origine des vésicules contenant les nanocristaux restent autant d'énigmes.

\section{Conclusion}

La plaque de Randall est un processus très fréquent a minima dont le principal déterminant est vraisemblablement la sursaturation interstitielle en phosphate de calcium.

\section{Déclaration de liens d'intérêts}

Les auteurs déclarent ne pas avoir de liens d'intérêts. 